\title{
Reaction mechanisms of methylene-blue degradation in three-dimensionally integrated micro-solution plasma
}

Tatsuru Shirafuji, Yodai Ishida, Ayano Nomura, Yui Hayashi and Motonobu Goto

\begin{tabular}{|c|l|}
\hline Citation & Japanese Journal of Applied Physics, 56(6S2); 06HF02 \\
\hline Issue Date & 2017-05-16 \\
\hline Type & Journal Article \\
\hline Textversion & Author \\
\hline Rights & $\begin{array}{l}\text { C } 2016 \text { The Japan Society of Applied Physics. The final publication is available at } \\
\text { https://doi.org/10.7567/JJAP.56.06HF02. } \\
\text { This is the accept manuscript version. Please cite only the published version. } \\
\text { 引用の際には出版社版をご確認ご利用ください。 }\end{array}$ \\
\hline DOI & \begin{tabular}{l} 
10.7567/JJAP.56.06HF02 \\
\hline
\end{tabular} \\
\hline
\end{tabular}

Self-Archiving by Author(s)

Placed on: Osaka City University

Tatsuru Shirafuji et al. 2017. Reaction mechanisms of methylene-blue degradation in three-dimensionally integrated micro-solution plasma. Japanese Journal of Applied Physics. 56 06HF02 


\title{
Reaction mechanisms of methylene-blue degradation in three-dimensionally integrated micro-solution plasma
}

\author{
Tatsuru Shirafuji*1, Yodai Ishida ${ }^{1}$, Ayano Nomura ${ }^{1}$, Yui Hayashi $^{2}$, and Motonobu Goto ${ }^{2}$ \\ ${ }^{1}$ Department of Physical Electronics and Informatics, Osaka City University, Osaka 558-8585, Japan \\ ${ }^{2}$ Department of Chemical Engineering, Nagoya University, Nagoya 464-8603, Japan
}

\begin{abstract}
We have performed matrix-assisted laser desorption ionization time-of-flight (MALDI-TOF) mass spectrometry (MS) on methylene-blue aqueous solutions treated with three-dimensionally integrated micro-solution plasma, in which we have acquired the time evolution of mass spectra as a function of treatment time. The time evolution of mass spectral peak intensities for major detected species has clearly indicated that the parent methylene-blue molecules are degraded through consecutive reactions. The primary reaction is the oxidation of the parent molecules. The oxidized species still have two benzene rings in the parent molecules. The secondary reactions are the separation of the oxidized species and the formation of compounds with one benzene ring. We have also performed the numerical fitting of the time evolution of the mass spectral peak intensities, the results of which have indicated that we must assume additional primary reactions before the primary oxidation for better agreement with experimental results.
\end{abstract}

\section{Introduction}

Solution plasma, which is low-temperature plasma in or in contact with an aqueous solution, can be applied to the direct treatment of liquid materials without the vaporization of the materials to be treated. The pioneering work on solution plasma has been reported between 2006 and 2008 by Baroch and coworkers. ${ }^{1-4)}$ One of the important applications of solution plasma is water treatment for degrading organic pollutants in water. Since there are many possible methods of generating plasma in contact with an aqueous solution, many researchers have reported various types of solution plasma systems. ${ }^{5-7)}$ Among them, the degradation of organic dyes such as methylene blue (MB) has frequently been used as a bench mark test for the original solution plasma system developed by each researcher. ${ }^{8-14)}$

The degradation of organic dyes is also important for reducing the environmental impact of the textile, leather, paper, plastic, food, cosmetic, and other industries. Many techniques other than those using solution plasma have been investigated to realize ideal degradation

*E-mail: sirafuji@elec.eng.osaka-cu.ac.jp 
processes. ${ }^{15-22)}$ In most cases, including plasma degradation processes, $\mathrm{OH}$ radicals are believed to play important roles in the degradation processes. However, there are few reports on detailed reaction mechanisms based on the analysis of reaction products, especially in the case of plasma degradation. Shirafuji et al. applied Fourier transform infrared (FTIR) spectroscopy to analyze reaction products. ${ }^{23)}$ Takenaka and Setsuhara applied X-ray photoelectron spectroscopy (XPS) together with FTIR. ${ }^{24)}$ Although FTIR and XPS are powerful tools for analyzing the overall chemical composition of a material, they do not give us information on molecules existing in a medium. On the other hand, mass spectrometry is one of the solutions for distinguishing molecules in a medium. Thus, we applied matrix-assisted laser desorption ionization time-of-flight mass spectrometry (MALDI-TOF MS) because it has a unique feature of suppressed fragmentation of detected species among various mass spectrometric techniques. $^{25)}$

In our previous paper, we proposed a possible reaction mechanism for MB degradation in solution plasma, which was deduced from data of MALDI-TOF MS on MB aqueous solutions treated with our novel solution plasma, which is three-dimensionally integrated microsolution plasma (3D IMSP), ${ }^{14,26,27)}$ the power efficiency of which for MB degradation was approximately 16-fold higher than that of the conventional SP generated between two tiny electrodes in an aqueous solution. ${ }^{13)}$ The deduced mechanism is depicted in Fig. 1, in which groups $\mathrm{A}, \mathrm{B}$, and $\mathrm{C}$ represent the parent $\mathrm{MB}$, primary products, and secondary products, respectively. Note that $m / z=312,192.5,174,112.5,110$, and 94 were not considered in our previous model, which will be discussed later.

However, the mechanism that we proposed was deduced from a few lines of evidence of mass spectra for MB aqueous solutions only before and after 30 min treatments. From the viewpoint of chemical engineering, our previously proposed model should be able to explain the time evolution of chemical species concentration. Thus, in this work, we have conducted MALDI-TOF MS as a function of plasma treatment time. We discuss the time evolution of mass spectral intensities for major detected species and verify the validity of our previously proposed model for MB degradation by solution plasma.

\section{Experimental procedure}

Details of our experimental setup for 3D IMSP were reported previously. ${ }^{13,14)}$ The liquid to be treated with 3D IMSP was MB aqueous solution with an MB concentration and a volume of $3.73 \mathrm{mg} / \mathrm{L}$ and $150 \mathrm{~mL}$, respectively. The temperature of the liquid was kept at $25^{\circ} \mathrm{C}$ during 3D IMSP treatments by circulating the liquid through a cooling device. The discharge gas was 


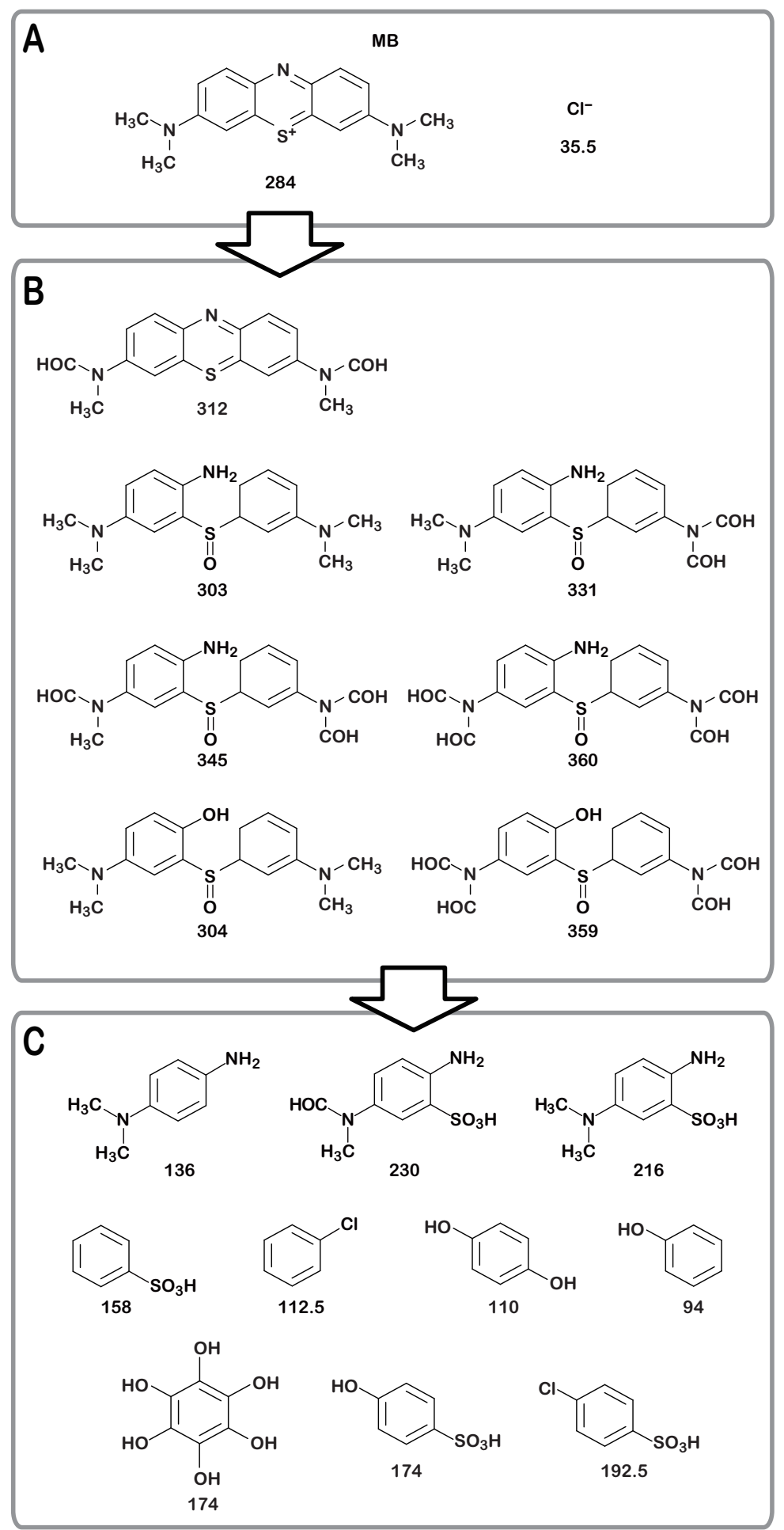

Fig. 1. Consecutive MB degradation mechanism based on our previous paper. ${ }^{26)}$

argon at a flow rate of $1.1 \mathrm{~L} / \mathrm{min}$. The applied voltage for generating plasma was a bipolar pulse voltage with an amplitude, a frequency, and a pulse width of $5 \mathrm{kV}, 20 \mathrm{kHz}$, and $2 \mu \mathrm{s}$, respectively. 
We prepared six samples, which were MB aqueous solutions treated with 3D IMSP for $0,10,20,30,40$, and $50 \mathrm{~min}$, and aquired their mass spectra. The mass spectrometry of the samples was conducted with a MALDI-TOF mass spectrometer (Bruker Daltonics Ultraflex III-ND TOF/TOF) equipped with a Nd:YAG laser (wavelength: $355 \mathrm{~nm}$ ) under the same measurement conditions for all the samples. We performed three measurements for each sample.

The sampling and enclosing of the aqueous solution before and after the treatment were carried out in a glove box filled with inert argon gas, because we observed the deterioration of the samples during 1 day air exposure while transferring them from our 3D IMSP reactor to the MALDI-TOF mass spectrometer. ${ }^{28)}$

The sample matrix for MALDI was 2,5-dihydroxybenzonic acid (DHB). DHB is known to produce mass spectral peaks at 137.3, 154.3, 177.3, 199.3, 273.4, 313.5, and 331.5. These mass spectral peaks may interfere with the peaks for the molecules that we are interested in. ${ }^{26)}$ In this case, we must appropriately interpret the time evolution of such mass spectral peaks, which will be discussed later.

In the case of MALDI-TOF MS, all the species can be detected as hydrogenated adducts owing to protonation, which means that a certain species $\mathrm{M}$ with a mass number of $\mathrm{m} / \mathrm{z}$ can be detected as mass spectral peaks for hydrogenated ones denoted by $\mathrm{M}: n \mathrm{H}$ at a mass number of $m / z+n$. The most abundant species are those with $n=1$ and $n=2 .{ }^{29,30)}$ However, this depends on the condition of MALDI, which is governed by various factors such as the type of matrix, analytes in the matrix, and laser wavelength and fluence. $n=3$ or higher is possible when the analyte is MB. ${ }^{31,32)}$ Thus, we focused on one or two species with similar time evolution characteristics.

\section{Results and discussion}

\subsection{MB degradation characteristics}

Figure 2(a) shows the spectra of the absorption coefficient of the aqueous solution treated with 3D IMSP. MB molecules have a broad absorption band centered at $665 \mathrm{~nm} .{ }^{33)}$ We can observe a typical absorption profile for MB in Fig. 2(a), and the absorption coefficient gradually decreases with increasing treatment time. The measured absorption coefficients can be converted to the absolute concentration of MB in the aqueous solution because we know the initial concentration of MB. Thus, we can calculate the time evolution of the MB concentration in the aqueous solution treated with 3D IMSP.

Figure 2(b) shows the time evolution of the MB concentration converted from the absorption spectra, in which we can observe that its decay characteristics can be expressed by a 

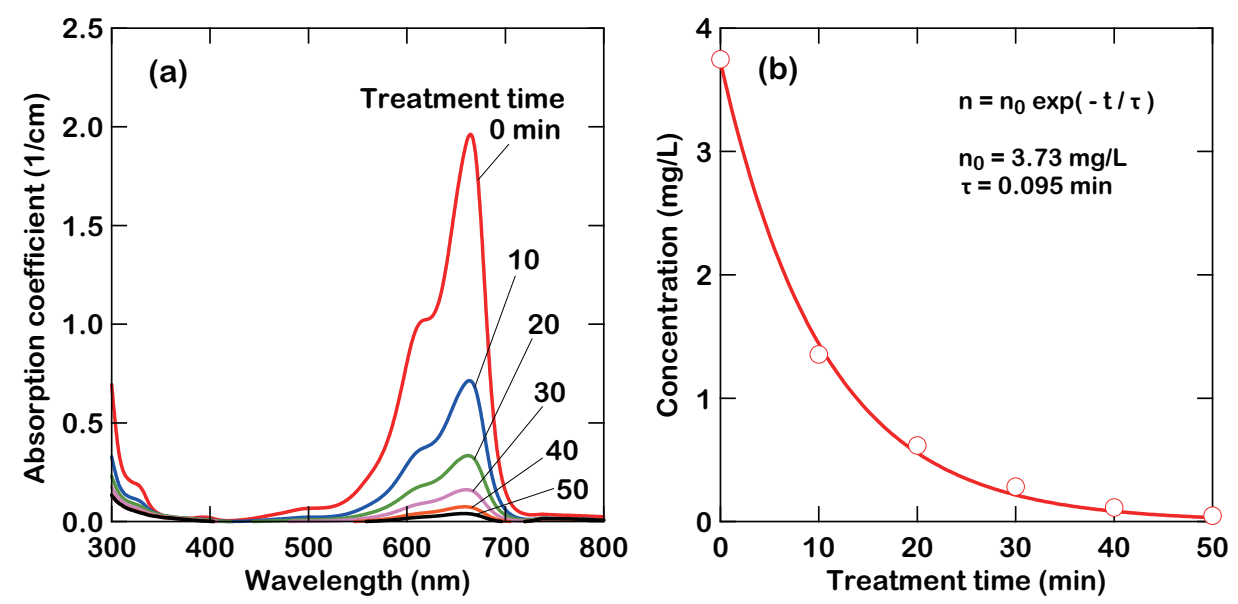

Fig. 2. (Color online) (a) Absorption spectra of MB aqueous solutions treated with 3D IMSP. (b) Time evolution of MB concentration in MB aqueous solutions treated with 3D IMSP.

single exponential function with a time constant of $\tau=0.095 \mathrm{~min}$. This means that the primary reactions for decomposing MB are first-order reactions. ${ }^{34)}$ Since the decomposition of $\mathrm{MB}$ is believed to be due to the attack of $\mathrm{OH}$ radicals on $\mathrm{MB}$ molecules, this result indicates that the 3D IMSP supplies a constant number density of $\mathrm{OH}$ radicals during the plasma treatment. We will later compare this characteristic to the time evolution of mass spectral peaks corresponding to MB.

\subsection{Time evolution of parent MB}

Figure 3 shows the time evolution of mass spectra for $m / z$ between 265 and 290. We can see major peaks in the two regions at approximately $m / z=284$ and 270 . The mass spectral peaks at approximately $m / z=284$ are assigned to the parent $\mathrm{MB}$, and those at $\mathrm{m} / z=285$, 286 , and 287 can be assigned to the hydrogenated MB denoted as MB: $n \mathrm{H}$. The mass spectral peaks at approximately $m / z=270$ are assigned to azure $\mathrm{B}(\mathrm{AB})$, the molecular structure of which is depicted in Fig. 4. ${ }^{35)}$ The mass spectral peaks at $m / z=271,272$, and 273 can be assigned to hydrogenated $\mathrm{AB}$ denoted as $\mathrm{AB}: n \mathrm{H}$. The formation of $\mathrm{AB}$ will be discussed later because $\mathrm{AB}$, which is not intentionally mixed in the nontreated aqueous solution, is not what we expect in the nontreated aqueous solution.

Figure 5(a) shows the time evolution of mass spectral intensities for $m / z=284$ and 285 corresponding to $\mathrm{MB}$ and $\mathrm{MB}: \mathrm{H}$, respectively. The time evolution characteristics of these intensities are well fitted with a single exponential function as indicated in the figure by solid lines. The time constant obtained by the fitting becomes $0.090 \mathrm{~min}$ for both $\mathrm{m} / z=284$ and 285, which shows good agreement with the time constant for MB degradation discussed in the 


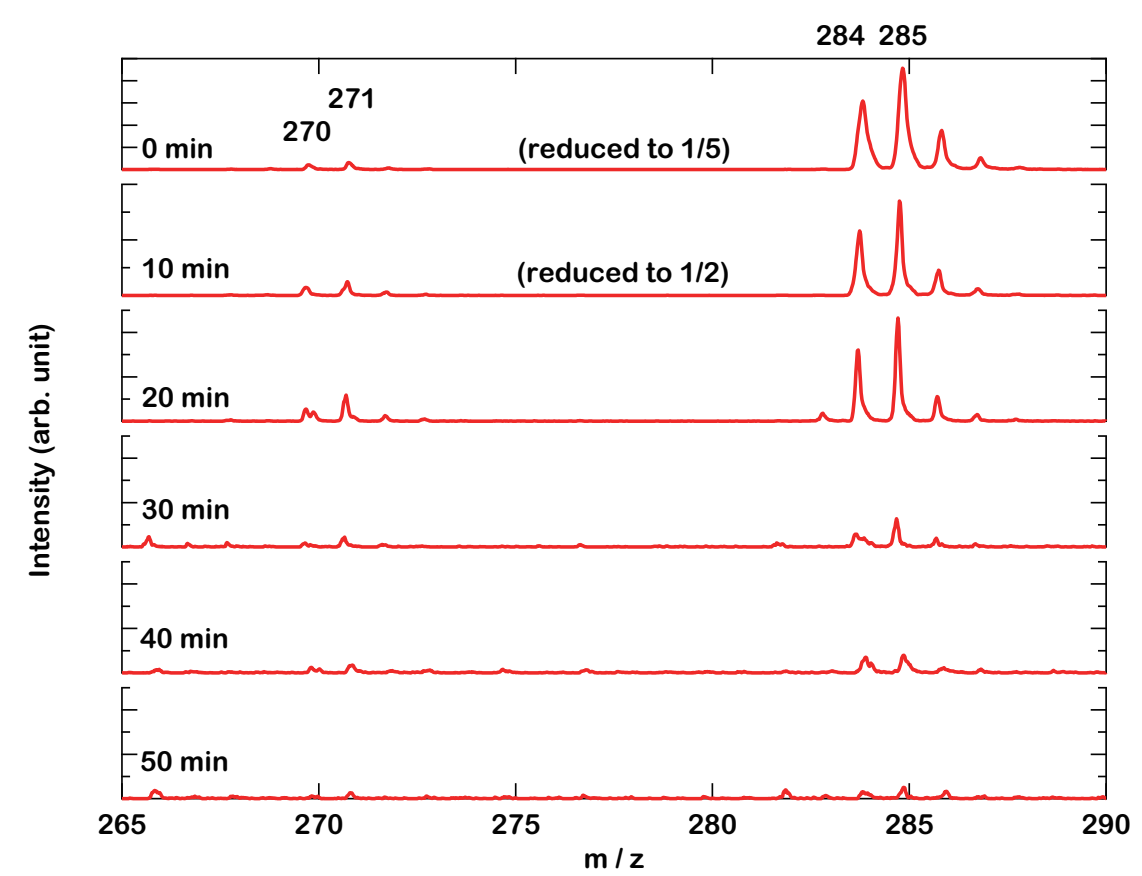

Fig. 3. (Color online) Mass spectra for $m / z=265-290$ of MB aqueous solutions treated with 3D IMSP.

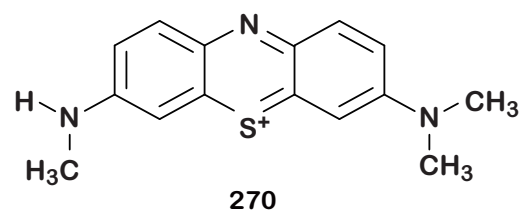

Fig. 4. Molecular structure of $\mathrm{AB}$.

previous subsection. This means that the sensitivity of MALDI-TOF MS was kept constant for all the measurements conducted in this work.

Figure 5(b) shows the time evolution of mass spectral intensities for $m / z=270$ and 271, which correspond to $\mathrm{AB}$ and $\mathrm{AB}: \mathrm{H}$, respectively. Although $\mathrm{AB}$ was not intentionally incorporated to the aqueous solution before the treatment, its intensity is maximum in the case of the nontreated sample. Since the conversion from $\mathrm{MB}$ to $\mathrm{AB}$ occurs with the weak oxidation of $\mathrm{MB},{ }^{36)}$ this result means that part of $\mathrm{MB}$ was modified to $\mathrm{AB}$ by air exposure even before the plasma treatment. The formation of $\mathrm{AB}$ in the nontreated $\mathrm{MB}$ aqueous solution makes discussion complex because the primary products of plasma treatment can be generated not only from $\mathrm{MB}$ but also from $\mathrm{AB}$. However, the absolute intensity of $\mathrm{AB}$ is as small as $1 / 10$ that of MB. Thus, we assume that most of the products generated by the primary reaction originate from MB. 

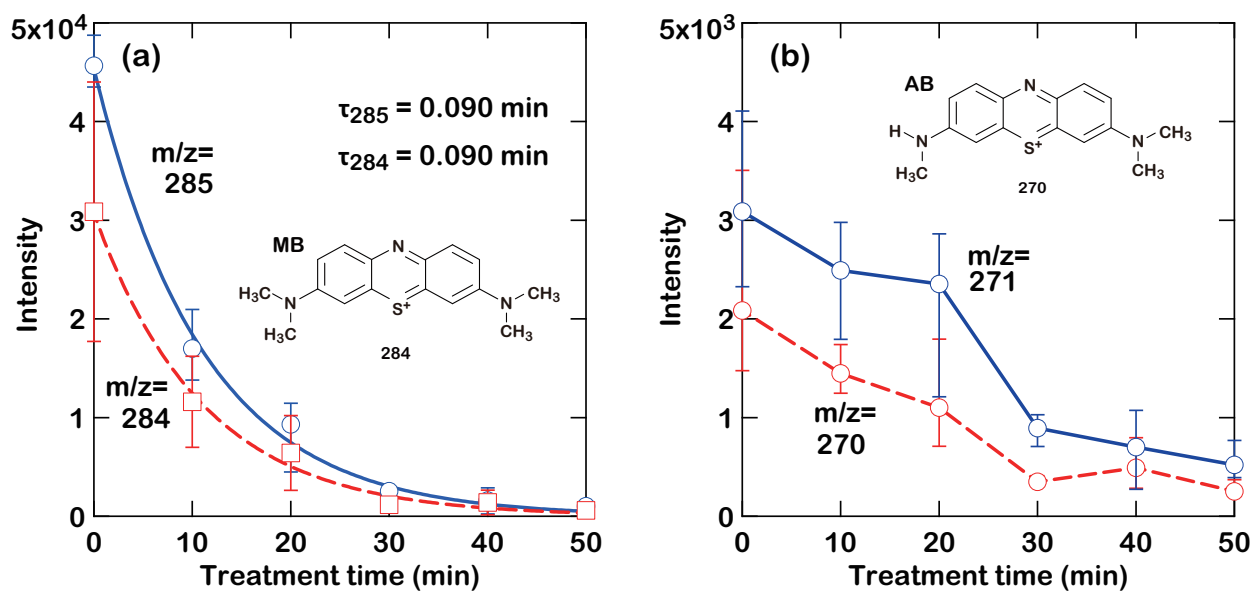

Fig. 5. (Color online) Time evolution of mass spectral intensities for (a) $m / z=284$ and 285 , which are assigned to $\mathrm{MB}$ and $\mathrm{MB}: \mathrm{H}$, and (b) $m / z=270$ and 271, which are assigned to $\mathrm{AB}$ and $\mathrm{AB}: \mathrm{H}$, respectively.

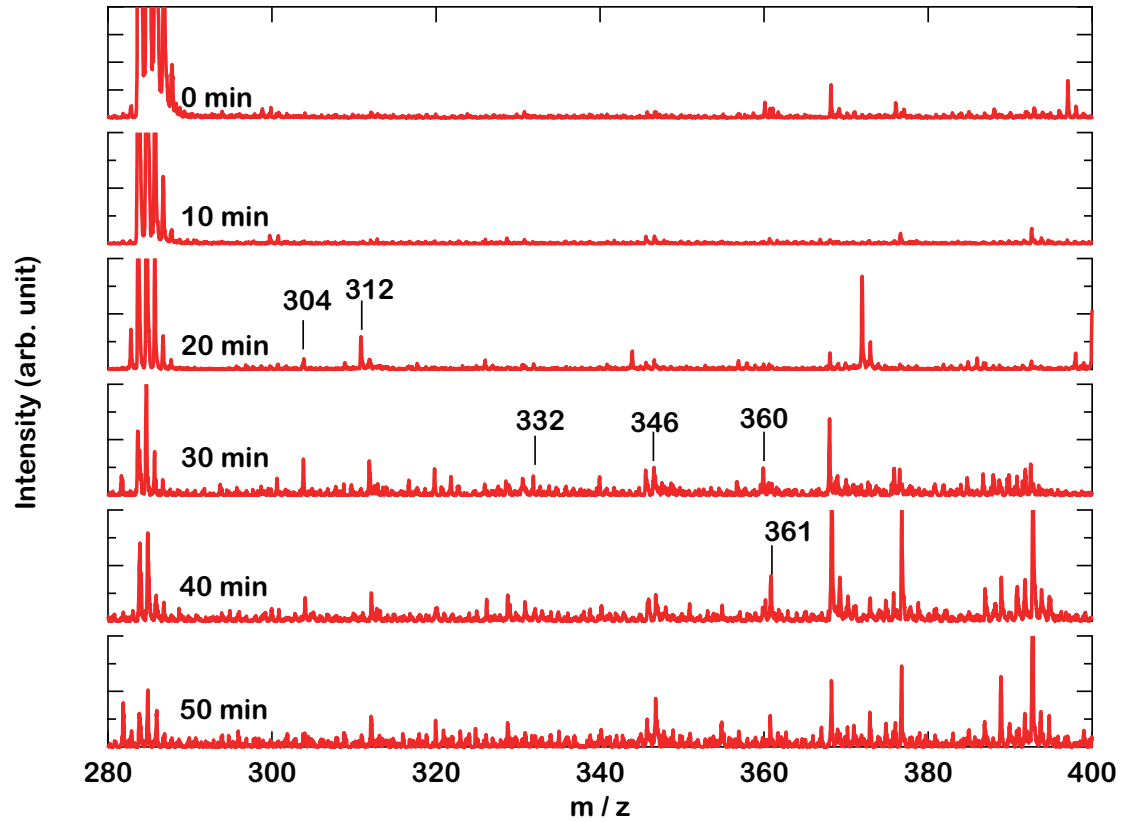

Fig. 6. (Color online) Mass spectra for $m / z=280-400$ of MB aqueous solutions treated with 3D IMSP.

\subsection{Time evolution of primary products}

In this subsection, we focus on mass numbers greater than $m / z=284$ of the parent MB. Figure 6 shows mass spectra for $m / z$ between 280 and 400. The mass spectral peaks at $m / z=304,312,332,346$, and 361, which correspond to the species in group B or their hydrogenated adducts, have their maximum intensities at approximetely $30 \mathrm{~min}$. Figure 7 shows the time evolution of mass spectral peak intensities for $m / z=304$ and 332, in which the peak intensities increase up to $30 \mathrm{~min}$ and decrease after that. These peaks are assigned 


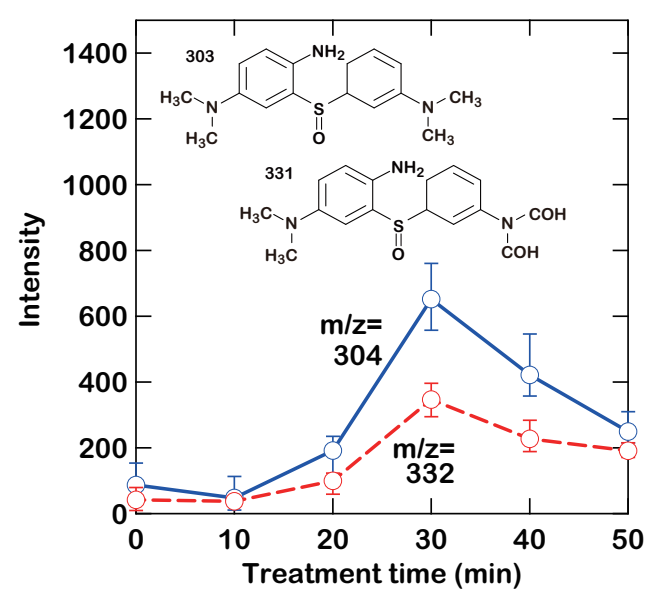

Fig. 7. (Color online) Time evolution of mass spectral intensities for (a) $m / z=304$ and (b) $m / z=332$, of which the convex characteristics indicate that these species are intermediate products in consecutive reactions.

to hydrogenated species of $m / z=303$ and $m / z=331$, which can be formed by modifying $\mathrm{C}-\mathrm{S}^{+}=\mathrm{C}$ or $\mathrm{C}-\mathrm{N}=\mathrm{C}$ between two benzene rings in an $\mathrm{MB}$ molecule and by modifying methyl groups at the edge of an MB molecule. The peaks at $m / z=312,346,360$, and 361, which can be formed by similar modifications, show similar tendencies, although they are not plotted in this figure.

These characteristics indicate that the species in group B are intermediate species in consecutive reactions as predicted in Fig. 1. Namely, the species in group B are not only produced from MB through primary reactions but also consumed through secondary reactions, which are presumably caused by the supply of abundant $\mathrm{OH}$ radicals.

These mechanisms are similar to those for the photocatalytic degradation of $\mathrm{MB},{ }^{15,37,38)}$ in which the middle part of an MB molecule is assumed to be attacked by $\mathrm{OH}$ radicals. However, interestingly, the formation of $m / z=312$, which is observed in the present work, has not been assumed in the photocatalytic degradation. Although it has not yet been clarified, this reaction path may be peculiar to solution plasma.

Note that the mass peak intensities for $m / z=363-390$ increase with treatment time. Some of the peaks can be assigned to the species formed by converting $\mathrm{COH}$ to $\mathrm{COOH}$ or $\mathrm{CH}$ on the benzene ring to $\mathrm{COH}$. Such species, however, have not been considered in the mechanism of photocatalytic degradation. ${ }^{15,37,38)}$ Although such species might also be peculiar to solution plasma, we cannot make appropriate conclusions because these species have a complex structure and their stability has not yet been clarified. 


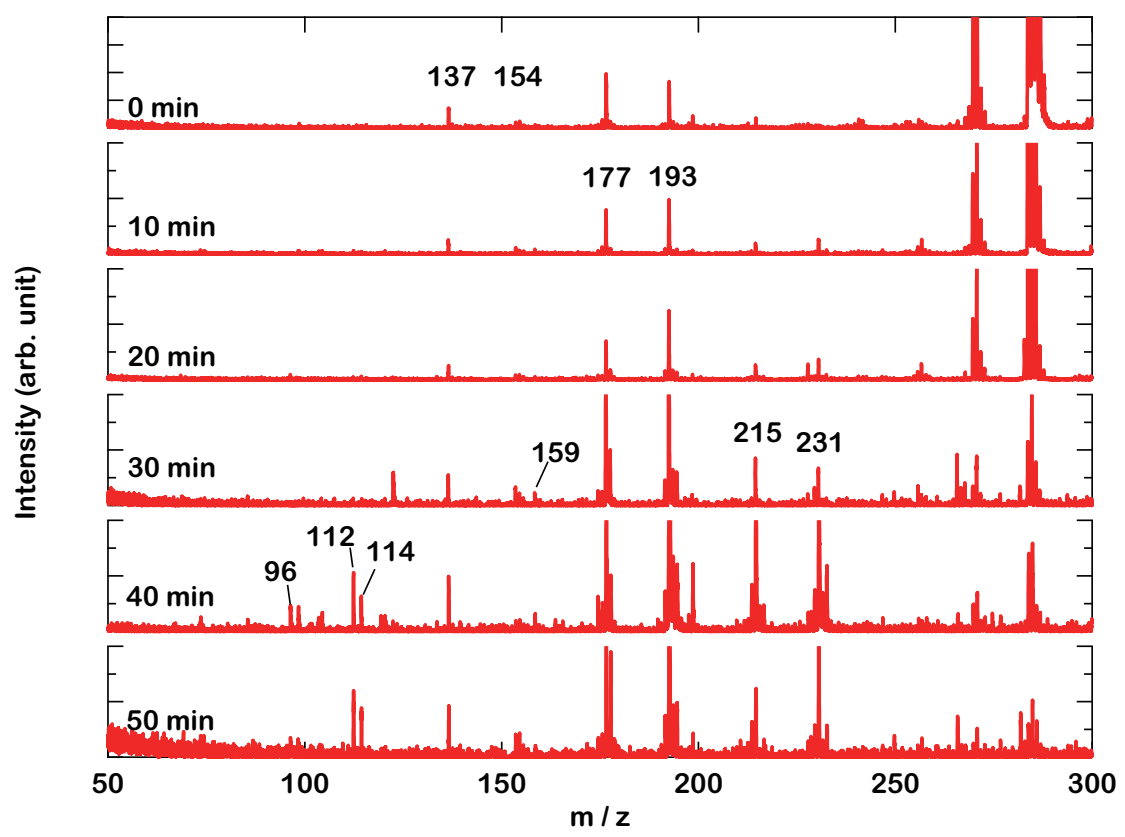

Fig. 8. (Color online) Mass spectra for $m / z=50-300$ of MB aqueous solutions treated with 3D IMSP.

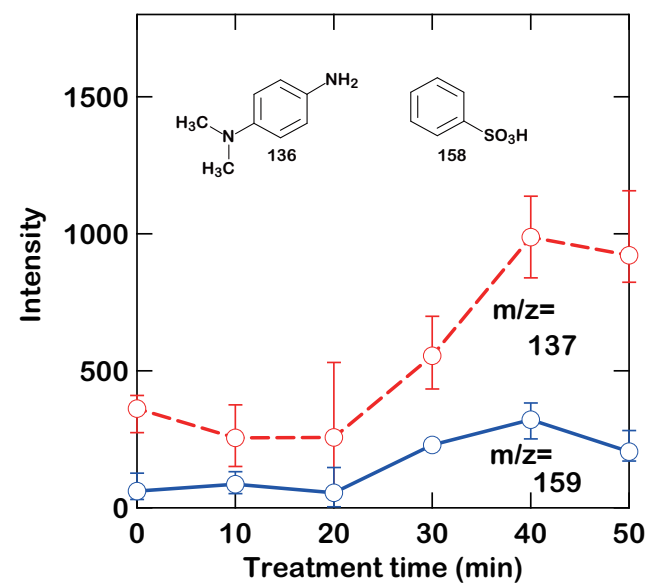

Fig. 9. (Color online) Time evolution of mass spectral intensities for (a) $\mathrm{m} / z=137$ and (b) $\mathrm{m} / \mathrm{z}=159$, the convex characteristics of which indicate that these species are intermediate products in consecutive reactions.

\subsection{Time evolution of secondary products}

In this subsection, we focus on mass numbers smaller than $m / z=284$ of the parent $\mathrm{MB}$, which corresponds to the species in group C in Fig. 1. Figure 8 shows mass spectra for $m / z$ between 50 and 300 . In contrast to the results discussed in the previous subsection, several mass spectral peaks show a monotonic increase in their intensity up to $50 \mathrm{~min}$. Figure 9 shows the time evolution of mass spectral peak intensities for $m / z=137$ and 159 . Their peak intensities do not show marked increases up to $20 \mathrm{~min}$. The peak intensity for $\mathrm{m} / z=137$ is 
relatively high owing to interference by one of the mass spectral peaks of the matrix DHB, as mentioned previously. After $20 \mathrm{~min}$, the peak intensities monotonically increase and slightly decrease at $50 \mathrm{~min}$. The peaks at $\mathrm{m} / \mathrm{z}=96,112,114,177,193,215$, and 231 were not discussed in our previous report. However, they can be formed by similar bond breaking, oxidation, and chlorine attachment processes and show similar tendencies, although they are not plotted in this figure. Possible species for these $m / z$ values are depicted in group C in Fig. 1 , in which the increment in $m / z$ due to protonation is taken into account.

The species in group $\mathrm{C}$ have only one benzene ring, which means that they are produced by breaking the chemical bonds connecting two benzene rings. Such species can be produced from both the parent MB in group A and the primary products in group B. However, their intensities do not show marked increases for the first 20 min at which MB markedly decomposed, indicating that they are not products of MB decomposition. The formation of these species in group B starts after a certain delay time, indicating that they are formed after the formation of a sufficient number of primary products in group B. These discussions lead to the conclusion that the consecutive reactions predicted in Fig. 1 surely occur during the plasma treatment.

\subsection{Numerical investigation of the model}

The results and discussion in the above subsections support our previously proposed $\mathrm{MB}$ degradation model composed of consecutive reactions from A to $\mathrm{C}$, which is shown in Fig. 1. If we assume such consecutive reactions, we can make a simple model to describe these reactions as

$$
\begin{aligned}
& \mathrm{A} \rightarrow \mathrm{B}, \\
& \mathrm{B} \rightarrow \mathrm{C},
\end{aligned}
$$

where A, B, and C indicate the species in groups A, B, and C, respectively. We do not distinguish the species in each group for simplicity because their time evolutions show similar tendencies. Rate equations for these reactions become ${ }^{34)}$

$$
\begin{aligned}
& \frac{d[\mathrm{~A}]}{d t}=-k_{1}[\mathrm{~A}], \\
& \frac{d[\mathrm{~B}]}{d t}=k_{1}[\mathrm{~A}]-k_{2}[\mathrm{~B}], \\
& \frac{d[\mathrm{C}]}{d t}=k_{2}[\mathrm{~B}],
\end{aligned}
$$




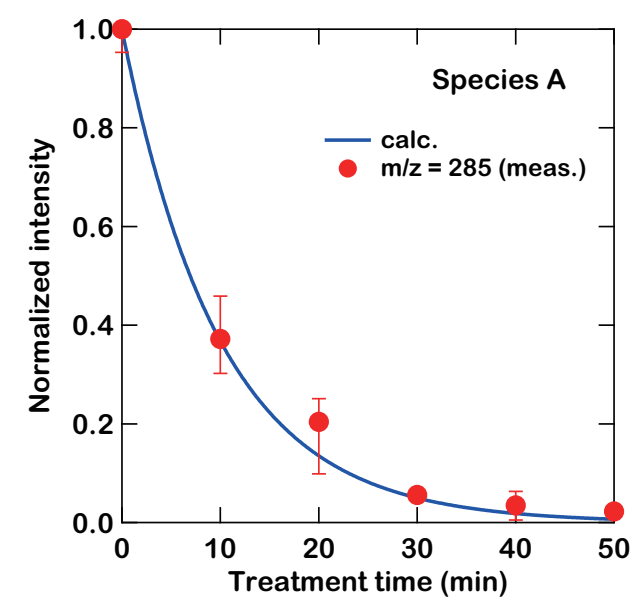

Fig. 10. (Color online) Fitting results of exponential decay characteristics of MB concentration in MB aqueous solutions treated with 3D IMSP.
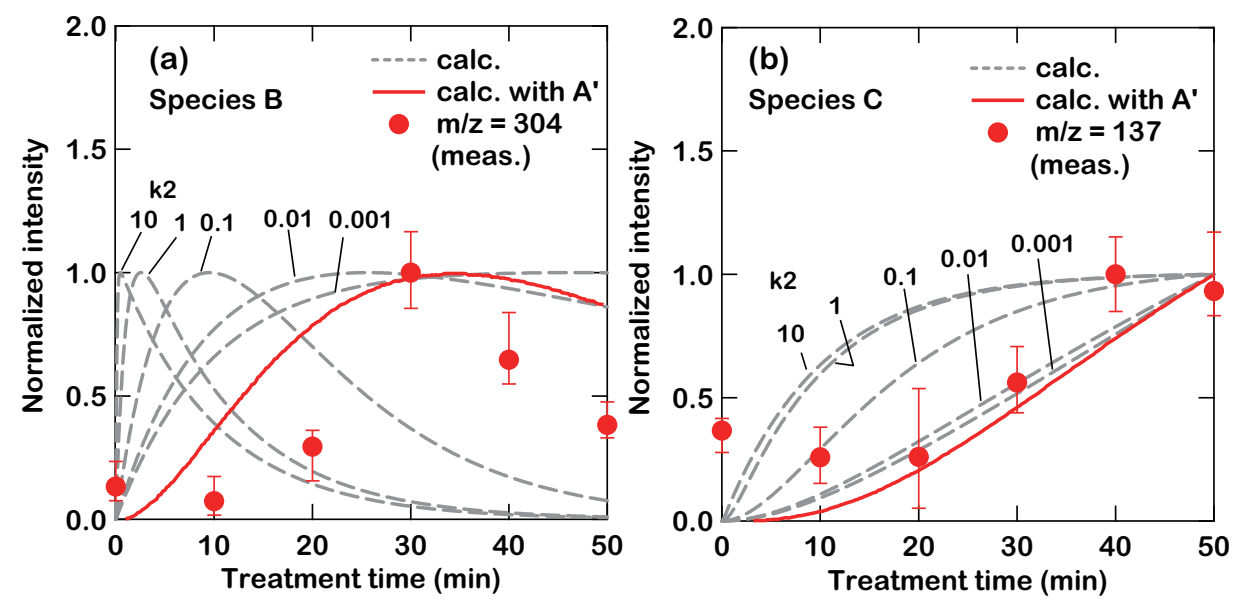

Fig. 11. (Color online) Time evolution of calculated relative concentrations of (a) species B and (b) species C. Dashed lines indicate the results calculated with reactions R1 and R2. Solid lines indicate those calculated with reactions $\mathrm{R} 1^{\prime}, \mathrm{R} 2^{\prime}$, and $\mathrm{R} 3^{\prime}$.

where [A], [B], and [C] are the concentrations of species A, B, and C, and $k_{1}$ and $k_{2}$ are rate coefficients for the reactions $\mathrm{R} 1$ and $\mathrm{R} 2$, respectively.

We have used this model to describe the behavior of MB $(m / z=285)$ in group A, $m / z=$ 304 in group $\mathrm{B}$, and $m / z=137$ in group C. $k_{1}$ is determined as 0.1 by fitting the exponential decay characteristics of MB, as shown in Fig. 10. $k_{2}$ should be selected to match the calculated behaviors of $\mathrm{B}$ and $\mathrm{C}$ with experimental results. We have calculated the time evolution of [B] and [C] using a wide $k_{2}$ range, and the obtained results are indicated by dashed lines in Figs. 11(a) and 11(b). As can be understood from these figures, we cannot reach good agreement between the calculated and experimental results, especially in the case of group B, 


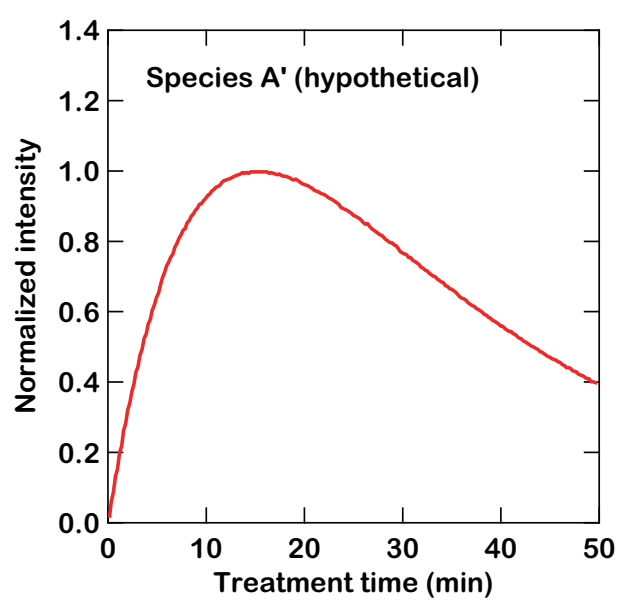

Fig. 12. (Color online) Time evolution of concentration of hypothetically introduced species $\mathrm{A}^{\prime}$.

even though we varied $k_{2}$ in a wide range.

The time evolution of the experimental results for group B seems to have some delay time, suggesting that additional primary reactions occur before producing the species of group B. Thus, we have supposed the following reactions:

$$
\begin{aligned}
& \mathrm{A} \rightarrow \mathrm{A}^{\prime}, \\
& \mathrm{A}^{\prime} \rightarrow \mathrm{B}, \\
& \mathrm{B} \rightarrow \mathrm{C} .
\end{aligned}
$$

We have fixed $k_{1}$ to 0.1 for $\mathrm{R} 1^{\prime}$, and adjusted $k_{2}$ and $k_{3}$ for $\mathrm{R} 2^{\prime}$ and $\mathrm{R} 3^{\prime}$, respectively. The solid lines in Figs. 11(a) and 11(b) show the best-fit results obtained using $k_{1}=0.1, k_{2}=0.04$, and $k_{3}=0.05$. As can be understood from these figures, solid lines show better agreement with experimental results, suggesting that additional primary reactions occur. However, the calculated results still show deviation from experimental results. This might be due to the fact that our present model is simplified on the basis of the assumption in which $\mathrm{OH}$ radicals as the oxidation agent are constantly supplied from plasma. To obtain a precise model, we must consider the effects of other possible oxidation agents such as $\mathrm{H}_{2} \mathrm{O}_{2}$, the concentration of which is not constant. $\mathrm{pH}$ may vary and affects the chemistry in the aqueous solution. We will make further discussion in the future by measuring $\mathrm{H}_{2} \mathrm{O}_{2}$ and $\mathrm{pH}$ in the plasma-treated MB aqueous solution.

In the modified model, we have the time evolution of the concentration of hypothetically introduced $\mathrm{A}^{\prime}$, which is shown in Fig. 12. Although we attempted to find the mass spectral peaks that show a time evolution similar to that of $\mathrm{A}^{\prime}$, we could not find such peaks. In the 
present calculation, we can perform the fitting of the time evolution of the relative concentration of the species, but information on the absolute concentration is not available except for the parent $\mathrm{MB}$. The missing information on $\mathrm{A}^{\prime}$ in our mass spectra is presumably due to the fast consumption of $\mathrm{A}^{\prime}$, which markedly reduces the steady-state concentration of $\mathrm{A}^{\prime}$.

\section{Conclusion}

We have performed MALDI-TOF MS on MB aqueous solutions treated with 3D IMSP for 0 to $50 \mathrm{~min}$. We have examined the time evolution of the mass spectral intensities for the detected species, which has indicated that MB degradation can be caused by consecutive decomposition reactions. The results obtained in this work support our previously proposed mechanism of MB degradation by solution plasma. Although we have attempted the numerical fitting of the time evolution of the mass spectral peak intensities, the calculated results have not shown sufficient agreement with the experimental results as long as we have assumed very simple consecutive reactions. By assuming additional primary reactions, the calculated results have shown better agreement with the experimental results, although the products of the assumed reactions have not been found in the mass spectra obtained in this work.

\section{Acknowledgment}

This work was partly supported by JSPS MEXT KAKENHI Grant Numbers $15 \mathrm{H} 03585$ and 15K13391. 


\section{References}

1) P. Baroch, T. Takeda, M. Oda, N. Saito, and O. Takai, 27th IEEE Int. Power Modulator Conf. / High Voltage Workshop, 2006, p. 482.

2) P. Baroch and N. Saito, IEEE Trans. Plasma Sci. 36, 1156 (2008).

3) T. Takeda, J. -S. Chang, T. Ishizaki, N. Saito, and O. Takai, IEEE Trans. Plasma Sci. 36, 1158 (2008).

4) O. Takai, Pure Appl. Chem. 80, 2003 (2008).

5) P. Bruggeman and C. Leys, J. Phys. D 42, 053001 (2009).

6) M. A. Malik, Plasma Chem. Plasma Process. 30, 21 (2010).

7) B. R. Locke, P. Lukes, and J.-L. Brisset, in Plasma Chemistry and Catalysis in Gases and Liquids, ed. V. I. Parvulescu, M. Magurenau, and P. Lukes (Wiley-VCH, Weinheim, 2012) Chap. 6.

8) K. Kitano, H. Aoki, and S. Hamaguchi, Jpn. J. Appl. Phys. 45, 8294 (2006).

9) T. Ishijima, H. Hotta, H. Sugai, and M. Sato, Appl. Phys. Lett. 91, 121501 (2007).

10) T. Maehara, I. Miyamoto, K. Kurokawa, Y. Hashimoto, A. Iwamae, M. Kuramoto, H. Yamashita, S. Mukasa, H. Toyota, S. Nomura, and A. Kawashima, Plasma Chem. Plasma Process. 28, 467 (2008).

11) M. Magureanu, D. Piroi, N. B. Mandache, and V. Parvulescu, J. Appl. Phys. 104, 103306 (2008).

12) Y. Takemura, N. Yamaguchi, and T. Hara, Jpn. J. Appl. Phys. 52, 056102 (2013).

13) T. Shirafuji and Y. Himeno, Jpn. J. Appl. Phys. 52, 11NE03 (2013).

14) T. Shirafuji, A. Nomura, and Y. Himeno, Plasma Chem. Plasma Process. 34, 523 (2014).

15) A. Houas, H. Lachheb, M. Ksibi, E. Elaloui, C. Guillard, and J.-M. Herrmann, Appl. Catal. B 31145 (2001).

16) H. Lachheb, E. Puzenat, A. Houas, M. Ksibi, E. Elaloui, C. Guillard, and J.-M. Hermann, Appl. Catal. B 39, 75 (2002).

17) R. J. Tayade, T. S. Natarajan, and H. C. Bajaj, Ind. Eng. Chem. Res. 48, 10262 (2009).

18) Y. Nosaka and A. Y. Nosaka, in Photocatalysis and Water Purification: From Fundamentals to Recent Applications, ed. P. Pichat (Wiley-VCH, Weinheim, 2013) Chap. 1.

19) D. Kobayashi, C. Honma, H. Matsumoto, T. Takahashi, Y. Shimada, C. Kuroda, K. Otake, and A. Shono, Jpn. J. Appl. Phys. 53, 07 KE03 (2014). 
20) M. Panizza, A. Barbucci, M. Delucchi, M. Carpanese, M. Cataldo-Hernández, and G. Cerisola, Sep. Purif. Technol. 118, 394 (2013).

21) G. Xia, Y. Lu, X. Gao, C. Gao, and H. Xu, Clean 43, 229 (2015).

22) A. Thiam, I. Sirés, J. A. Garrido, R. M. Rodríguez, and E. Brillas, J. Hazardous Mater. 290, 34 (2015).

23) T. Shirafuji, J. Hieda, O. Takai, N. Saito, T. Morita, O. Sakai, and K. Tachibana, Proc. IEEE Region 10 Conf., 2010, p. 1938.

24) K. Takenaka and Y. Setsuhara, Jpn. J. Appl. Phys. 52, 11 NE04 (2013).

25) F. Hillenkamp, T. W. Jaskolla, and M. Karas, in The MALDI Process and Method (Wiley-VCH, Weinheim, 2014) 2nd ed., Chap. 1.

26) T. Shirafuji, A. Nomura, Y. Hayashi, K. Tanaka, and M. Goto, Jpn. J. Appl. Phys. 55, 01AH02 (2016).

27) T. Shirafuji and A. Nakamura, Trans. Mater. Res. Soc. Jpn. 38321 (2013).

28) A. Nomura, Y. Himeno, Y. Hayashi, K. Tanaka, M. Goto, and T. Shirafuji, 15th IUMRS Int. Conf. Asia, 2014, D2-O26-009.

29) M. Karas, M. Clückmann, and J. Schäfer, J. Mass Spectrom. 35, 1 (2000).

30) R. Zenobi and R. Knochenmuss, Mass Spectrom. Rev. 17, 337 (1998).

31) M. V. Kosevich, V. V. Chagovets, I. V. Shmigol, S. V. Snegir, O. A. Boryak, V. V. Orlov, V. S. Shelkovsky, V. A. Pokrovskiy, and A. Gomory, J. Mass Spectrom. 43, 1402 (2008).

32) M. V. Kosevich, O. A. Boryak, V. V. Orlov, V. S. Shelkovsky, V. V. Chagovets, S. G. Stepanian, V. A. Karachevtsev, and L. Adamowicz, J. Mass Spectrom. 41, 113 (2006).

33) J. Cenens and R. A. Schoonheydt, Clays Clay Miner. 36, 214 (1988).

34) D. Ball, J. Chem. Educ. 75, 917 (1998).

35) A. Y. Khan and G. S. Kumar, Spectrochim. Acta, Part A 152, 417 (2016).

36) W. C. Holmes and B. W. French, Stain Technol. 1, 17 (1926).

37) A. Chithambararaj, N. S. Sanjini, A. C. Bose, and S. Velmathi, Catal. Sci. Technol. 3, 1405 (2013).

38) R. G. Chaudhuri and S. Paria, Dalton Trans. 43, 5526 (2014). 